Supporting Information

\title{
Near-Infrared Polymeric Nanoparticles with High Content of Cyanine for Bimodal Imaging and Photothermal Therapy
}

Wenhai Lin, ${ }^{\dagger}{ }^{\ddagger}$ Yang Li, ${ }^{\S}$ Wei Zhang,${ }^{\dagger}+$ Shi Liu,${ }^{\dagger}$ Zhigang Xie ${ }^{*}{ }^{\dagger}$ and Xiabin Jing ${ }^{\dagger}$

${ }^{\dagger}$ State Key Laboratory of Polymer Physics and Chemistry, Changchun Institute of Applied Chemistry, Chinese Academy of Sciences, 5625 Renmin Street, Changchun, Jilin 130022, P. R. China

University of Chinese Academy of Sciences, Beijing 100049, P. R. China

${ }^{\S}$ College of Environmental and Chemical Engineering, Yanshan University, Qinhuangdao 060004, P.R. China

\section{Corresponding Author}

*Zhigang Xie

E-mail: xiez@ciac.ac.cn 
Table S1. Formulation of CyP@PEG-PLA NPs

\begin{tabular}{|c|c|c|c|c|c|}
\hline Entry & $\begin{array}{c}\text { PEG-PLA/CyP } \\
(\mathrm{w} / \mathrm{w})\end{array}$ & $\begin{array}{c}\text { Loading } \\
\text { content }(\%)^{[\mathrm{a}]}\end{array}$ & $\begin{array}{c}\text { Loading } \\
\text { efficiency }(\%)^{[b]}\end{array}$ & $\begin{array}{l}\text { Diameter } \\
(\mathrm{nm})^{[\mathrm{c}]}\end{array}$ & $\operatorname{PdI}{ }^{[\mathrm{c}]}$ \\
\hline 1 & $1: 1$ & $44.2(28.1)^{[\mathrm{d}]}$ & 79.3 & $94.7 \pm 2.9$ & $0.152 \pm 0.004$ \\
\hline 2 & $1: 5$ & $77.4(49.1)$ & 68.4 & $88.2 \pm 4.3$ & $0.352 \pm 0.017$ \\
\hline 3 & $1: 10$ & $83.6(53.1)$ & 51.0 & $102.3 \pm 5.9$ & $0.386 \pm 0.035$ \\
\hline
\end{tabular}

[a] Loading content (wt \%) = the CyP amount in the micelles/the amount of micelles; [b] Loading efficiency (wt \%) = the CyP amount in the micelles/the drug feeding; [c] Diameter and PdI were measured by DLS; [d] CyCOOH loading content in the micelles (wt \%)

Table S2. The stability of CyP@PEG-PLA NPs (44.2\%)

\begin{tabular}{|c|c|c|}
\hline Sample & Diameter $(\mathrm{nm})^{[\mathrm{a}]}$ & PdI \\
\hline $\begin{array}{c}\text { CyP@PEG-PLA NPs } \\
(44.2 \%)^{[\mathrm{b}]}\end{array}$ & $94.7 \pm 2.9$ & $0.152 \pm 0.004$ \\
\hline $\begin{array}{c}\text { NPs in normal saline for } \\
\text { half a year }\end{array}$ & $94.4 \pm 0.8$ & $0.169 \pm 0.017$ \\
\hline $\begin{array}{c}\text { NPs in PBS with } 10 \% \\
\text { FBS for } 24 \mathrm{~h}\end{array}$ & $101.7 \pm 4.0$ & $0.196 \pm 0.026$ \\
\hline
\end{tabular}

[a] Diameter and PdI were measured by DLS; [b] Loading content (wt \%) 

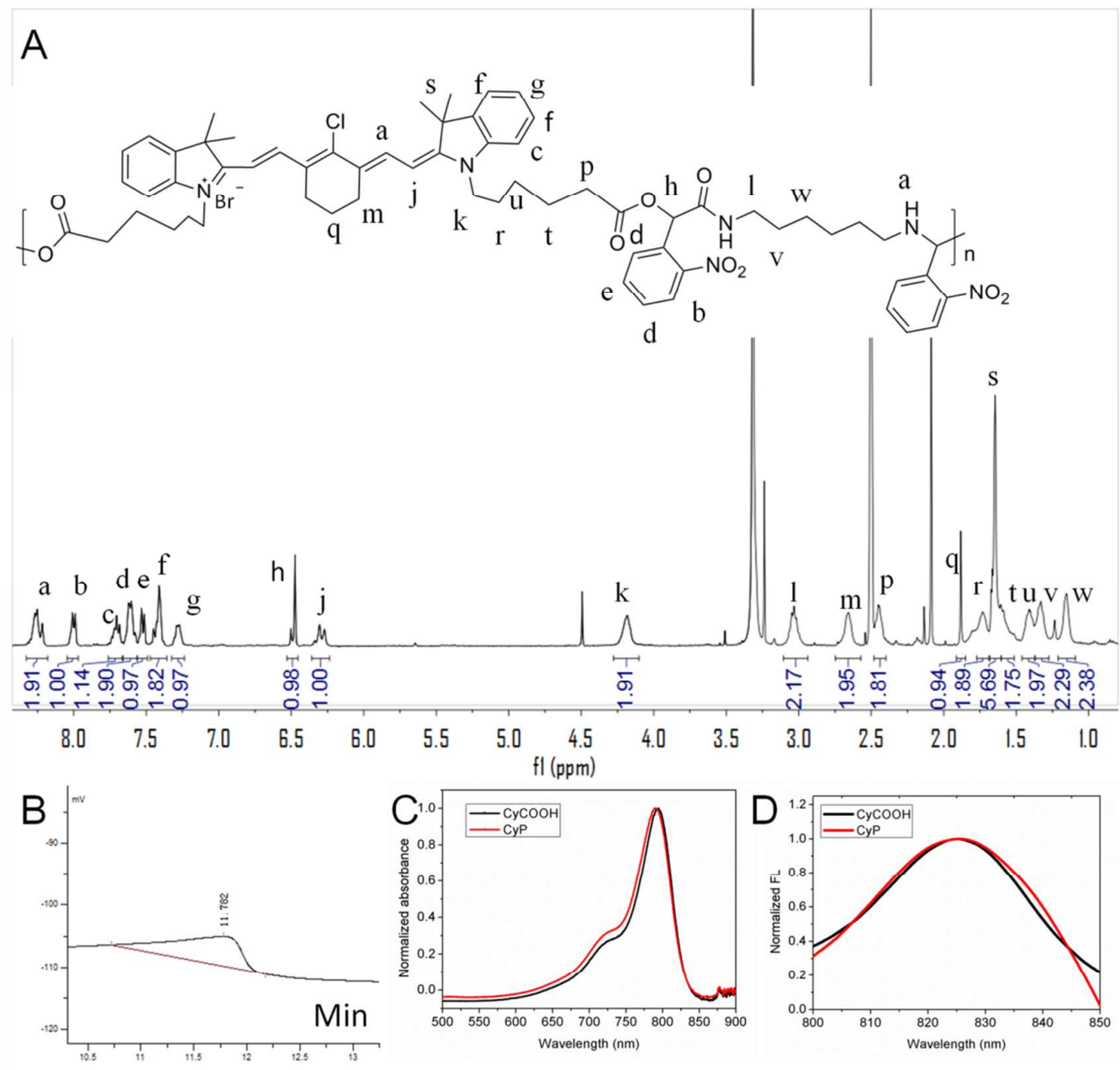

Figure S1. A) ${ }^{1} \mathrm{H}$ NMR characterization and B) The SEC trace of CyP. C) the UV-vis-NIR absorption spectra and D) The emission spectra of $\mathrm{CyCOOH}$ and $\mathrm{CyP}$. 


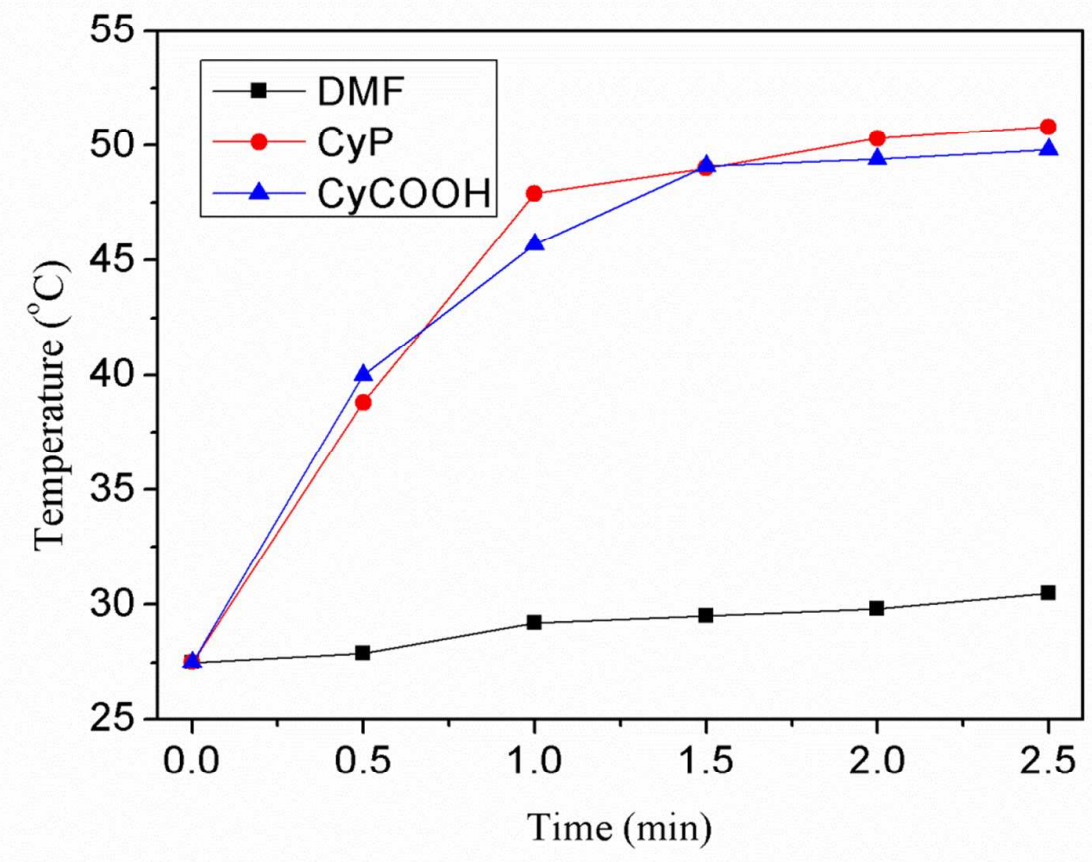

Figure S2. Heating curves of $\mathrm{CyP}$ and $\mathrm{CyCOOH}$ under NIR laser irradiation $(2 \mathrm{~W}$ $\left.\mathrm{cm}^{-2}\right)$ at the same concentration of $\mathrm{CyCOOH}\left(30 \mu \mathrm{gL}^{-1}\right)$.
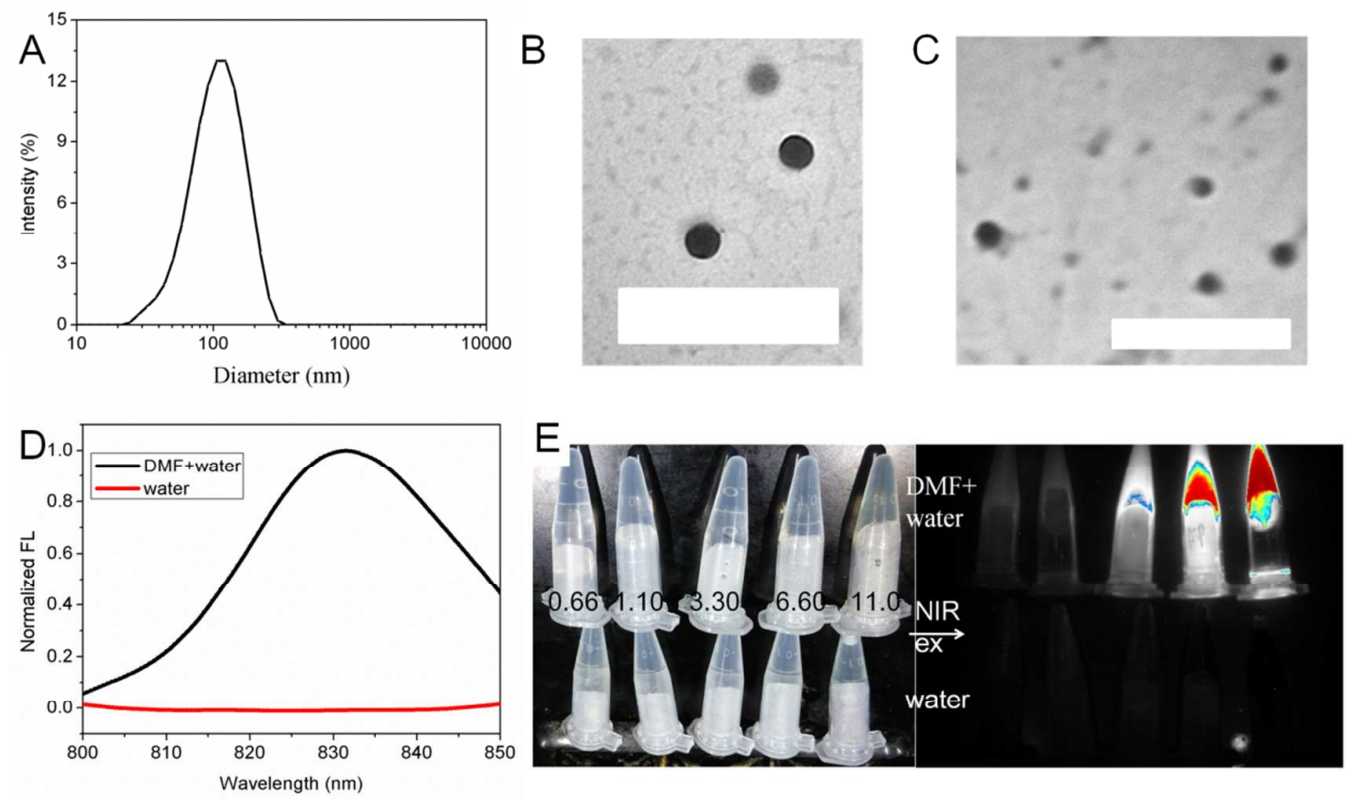

Figure S3. A) The diameter of CyP@PEG-PLA NPs measured by DLS. B) and C)

The TEM images of Cyp@PEG-PLA NPs (44.2 \% and $83.6 \%$ ). Scale bar, 500 nm. D) 
The emission spectra of CyP@PEG-PLA in water and in DMF+water (v/v, 1:1). E) The NIRF of CyP@PEG-PLA in water and in DMF+water $(\mathrm{v} / \mathrm{v}, 1: 1)$ at different concentrations. Unit, $\mu \mathrm{g} \mathrm{mL} \mathrm{m}^{-1}$.
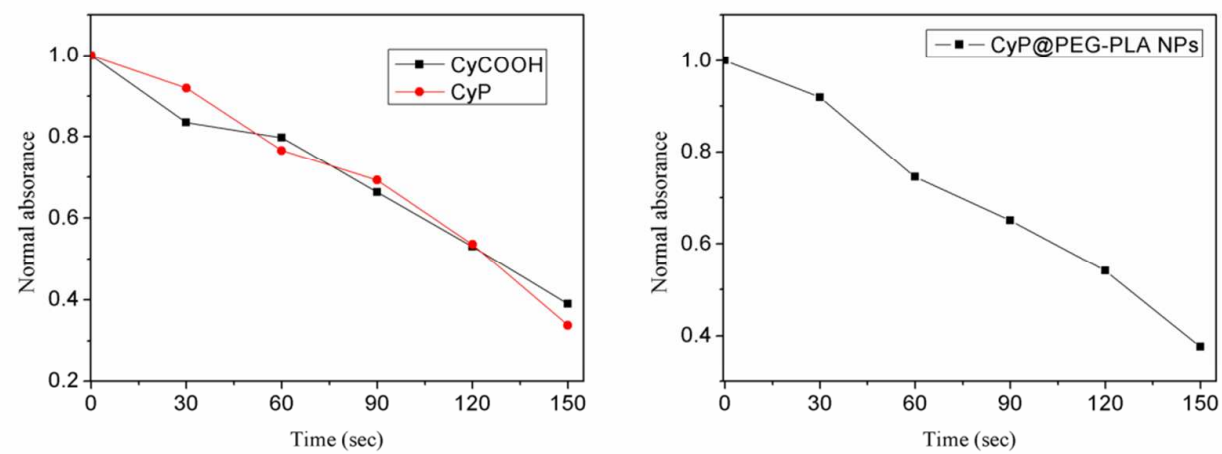

Figure S4. The stability of $\mathrm{CyCOOH}$ and $\mathrm{CyP}$ in DMF and CyP@PEG-PLA NPs in water with the $808-\mathrm{nm}$ laser irradiation $\left(2 \mathrm{~W} \mathrm{~cm}^{-2}\right)$ for 150 seconds.

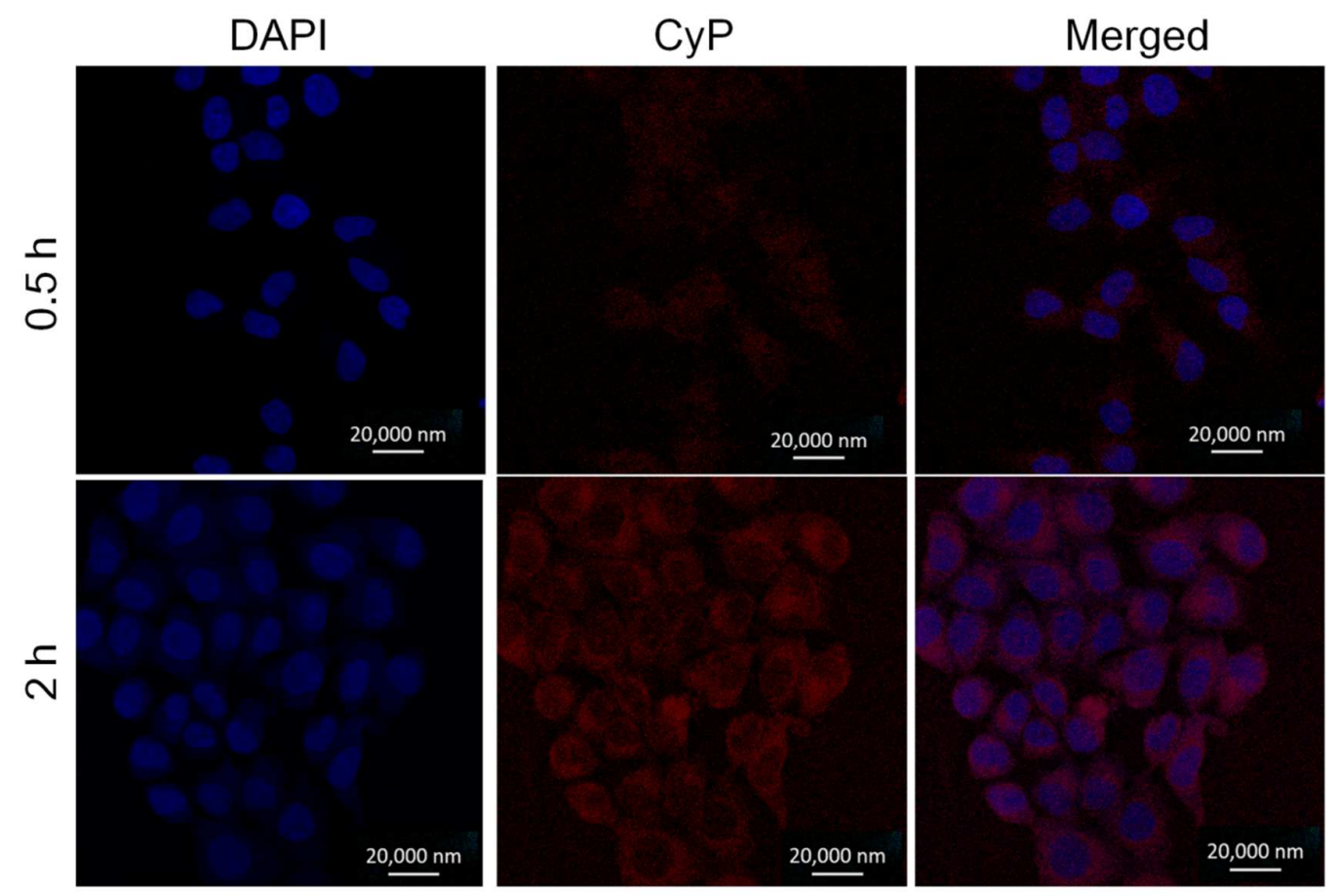

Figure S5. Representative CLSM images of HeLa cells incubated with CyP@PEG-PLA NPs for 0.5 h and 2 h. For each panel, the images from left to right 
show cell nuclei by DAPI (blue), CyP fluorescence in cells (red) and overlays of both images.
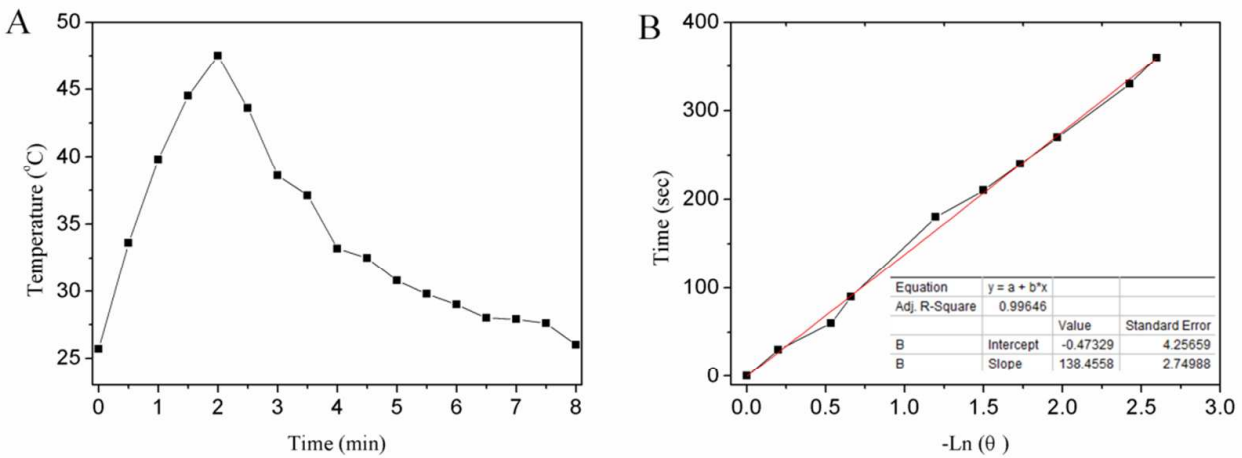

Figure S6. A) The photothermal response of CyP@PEG-PLA NPs in water (50 $\mu \mathrm{g} / \mathrm{mL})$ with laser irradiation $\left(808 \mathrm{~nm}, 1.5 \mathrm{~W} / \mathrm{cm}^{2}, 2 \mathrm{~min}\right)$ and then the laser was shut off. B) Linear time data versus $-\ln \theta$ obtained from the cooling period of (A).

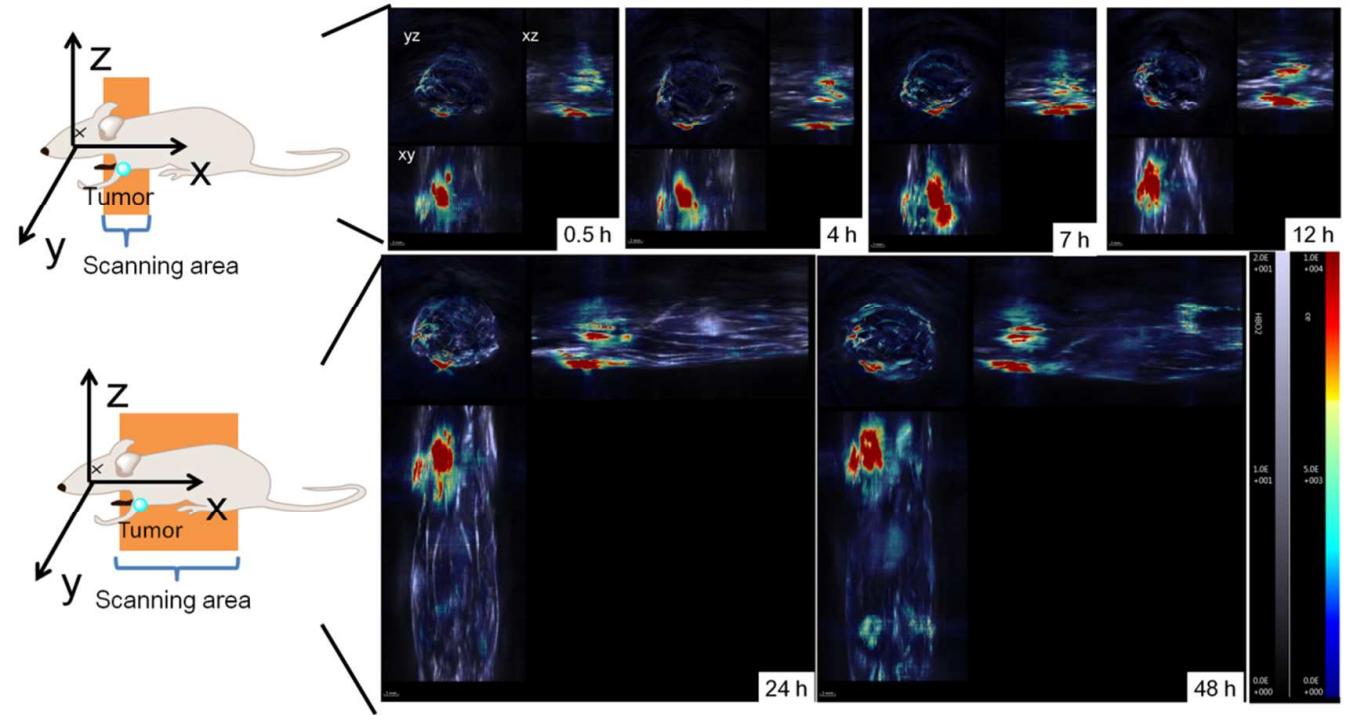

Figure S7. PA imaging of the HeLa-tumor-bearing mouse injected with CyP@PEG-PLA NPs $\left(100 \mu \mathrm{g} \mathrm{mL} \mathrm{L}^{-1}, 100 \mu \mathrm{L}\right)$ intratumorally. 

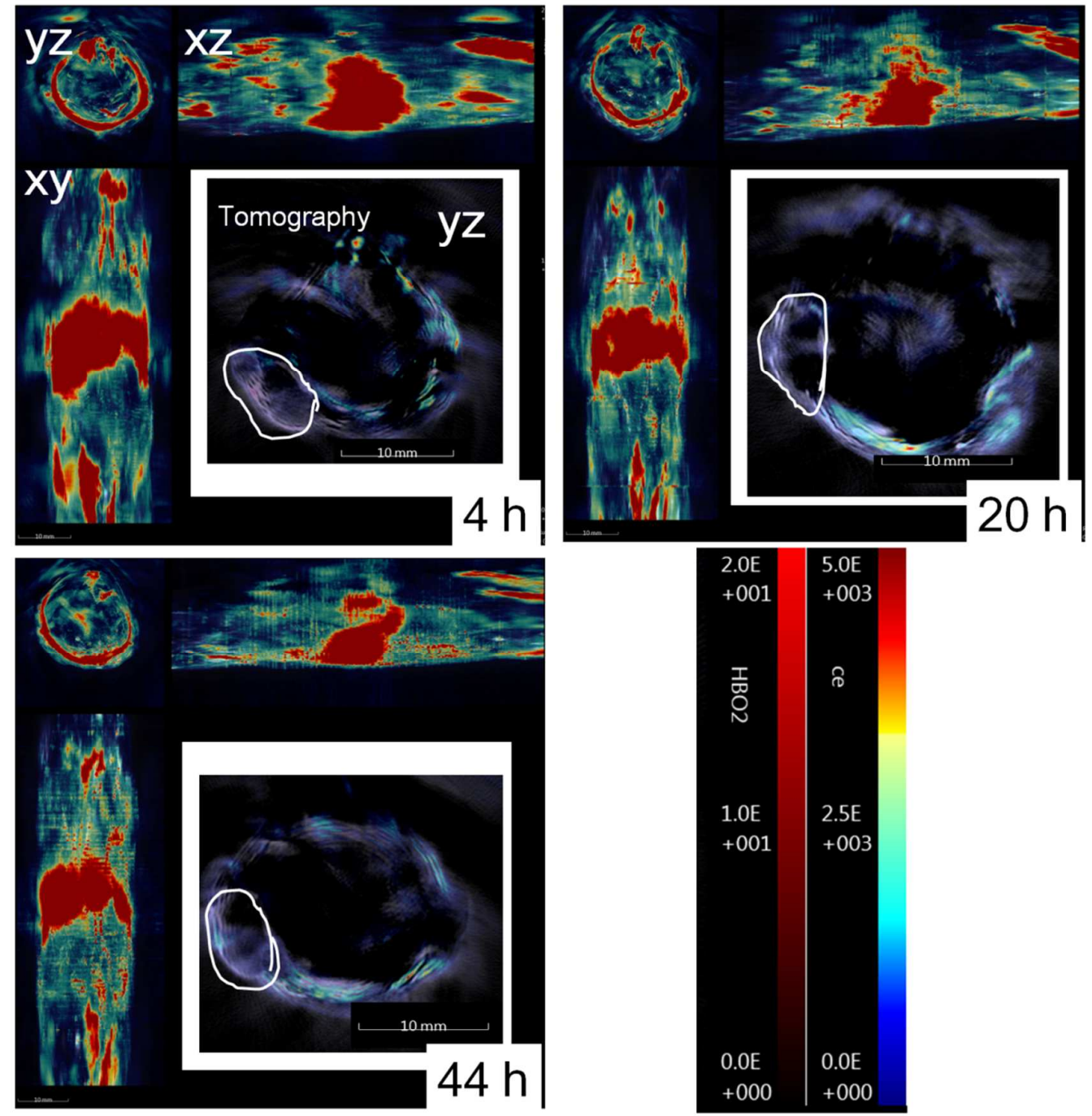

Figure S8. PA imaging of the HeLa-tumor-bearing mouse injected with CyP@PEG-PLA NPs intravenously. The area of white circle is the tumor. 

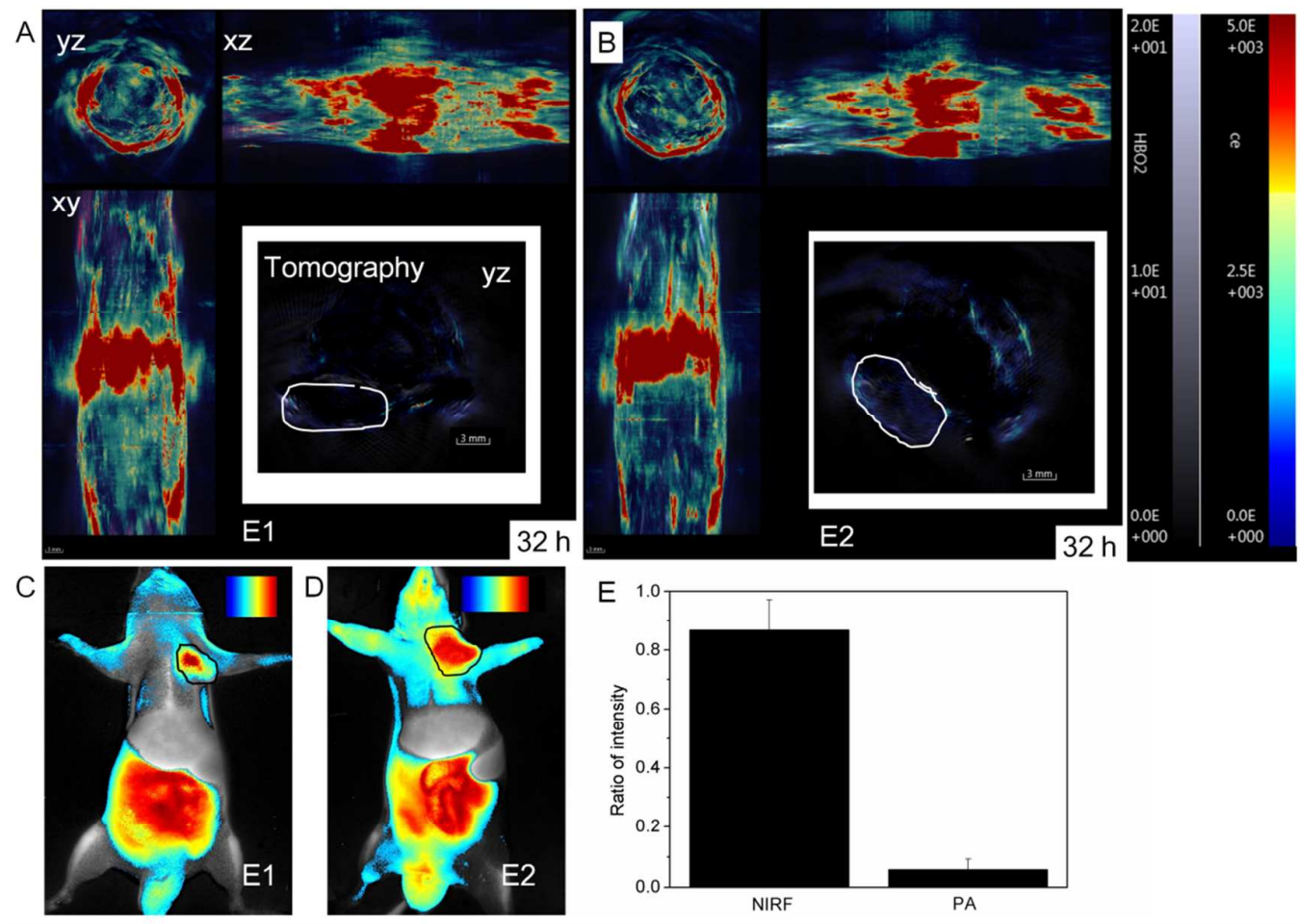

Figure S9. A) and B) PA imaging of two HeLa-tumor-bearing mouse injected with CyP@PEG-PLA NPs intravenously after 32 h. C) and D) NIRF imaging of two HeLa-tumor-bearing mouse injected with CyP@PEG-PLA intravenously after $32 \mathrm{~h}$. $\mathrm{E} 1$ and $\mathrm{E} 2$ is the serial number of the mice. E) The ratio between the intensities (NIRF and PA) in tumor and in liver in $32 \mathrm{~h}$. The area of white and black circle is the tumor.
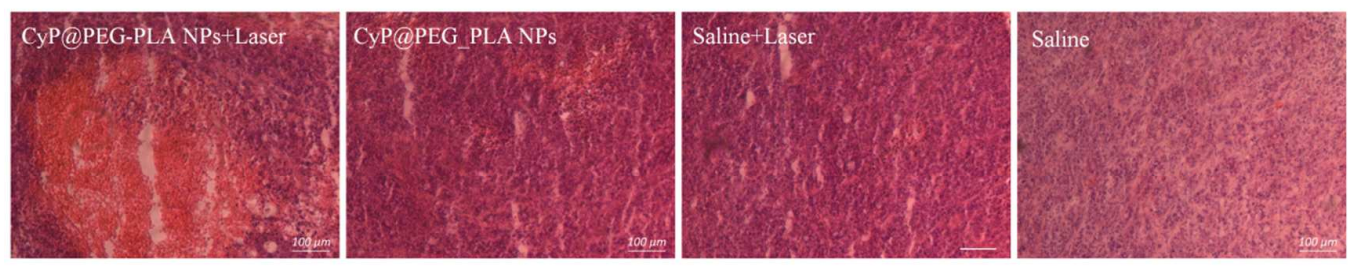

Figure S10. The H\&E staining of tumor tissues at the 12th day after treatment of various samples. The scale bar, $100 \mu \mathrm{m}$. 

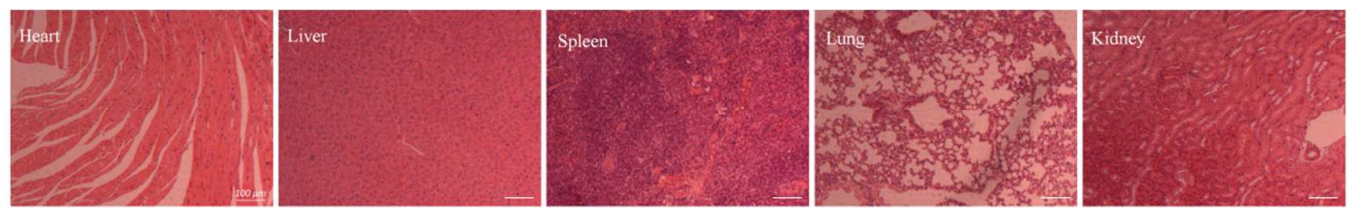

Figure S11. The H\&E staining of heart, liver, spleen, lung and kidney at the 12th day after treatment of CyP@PEG-PLA NPs. The scale bar, $100 \mu \mathrm{m}$. 